\section{P223a INCREASING THE OUALITY OF COPD CASE FINDING, DIAGNOSIS AND MANAGEMENT THROUGH A PRIMARY CARE FINANCIAL INCENTIVE SCHEME IN INNER LONDON}

doi:10.1136/thoraxjnl-2011-201054c.223a

${ }^{1} \mathrm{M}$ Calonge Contreras, ${ }^{1} \mathrm{~J}$ Billett, ${ }^{2} \mathrm{~L}$ Restrick, ${ }^{3} \mathrm{~K}$ Sennett, ${ }^{4} \mathrm{C}$ Cooper, ${ }^{2} \mathrm{M}$ Stern. ${ }^{1} \mathrm{NHS}$ North Central London, London, UK; ${ }^{2}$ Whittington Health, London, UK; ${ }^{3}$ Killick Street Health Centre, London, UK; ${ }^{4}$ St John's Way Medical Centre, London, UK

Introduction and Objectives COPD is a major cause of mortality/ morbidity in high smoking prevalence Primary Care Trusts (PCTs). Our PCT expected COPD prevalence $(3.7 \%)$ is therefore high but recorded prevalence (2009/2010) was $1.4 \%$, suggesting large numbers of undiagnosed patients. COPD, as the 2nd commonest cause of emergency admission locally, is one of the most costly diseases for secondary care. Local research (Bastin et al, $2010^{1}$ ) shows that, while most patients admitted for the first time with acute exacerbations of COPD have severe disease, there is no prior diagnosis in $\sim 1 / 3$ cases. A COPD Local Enhanced Service (LES) was developed, to incentivise practices to proactively identify, diagnose and manage COPD patients using evidence-based interventions.

Methods All GP practices in were invited to participate in the COPD LES. Key elements included number of case finding spirometries performed in smokers/ex-smokers $=35 \mathrm{y}$, and provision of interventions (pulmonary rehabilitation (PR) referral, self-management, oxygen auditing) with regular reviews/assessments. Primary outcomes were the number of new COPD diagnoses, a change in the gap between recorded and estimated COPD prevalence and number of non-elective hospital admissions. Data were extracted from the PCT GP dataset, OMAS (diagnosed prevalence), APHO COPDprevalence model (expected prevalence) and Secondary Users Services (hospital admission data).

Results $37 / 38$ (97\%) GP practices signed up to provide the LES. Between April 2010 and May 2011, 1807 case finding spirometries were performed resulting in an estimated 477 new COPD diagnoses, significantly reducing the undiagnosed COPD prevalence by $0.2 \%$ $(p<0.05)$. Compared to the same period in 2009, referrals to PR increased from 78 to $119(52 \%)$ in the first 6/12. Audits of oxygen therapy identified ongoing unnecessary payment in 52 patients (47 $\mathrm{died} / \mathrm{moved}$, five patients no longer required oxygen). Twenty-nine patients on LTOT had not been reviewed and were subsequently referred. The LES impact on the rate of emergency admissions for COPD remains unclear.

Conclusions One year evaluation demonstrates the COPD-LES is an effective strategy to improve case finding and diagnosis of COPD, improve PR referrals and rationalise oxygen prescribing. Ongoing audit of COPD emergency admissions will determine whether the LES achieves its objective.

Abstract P223a Table 1 Changes between recorded and expected prevalence of COPD, persons aged 16+, 2009-2011 (OOF)

\begin{tabular}{lllllll}
\hline & \multicolumn{2}{l}{ Recorded prevalence } & & \multirow{2}{*}{$\begin{array}{c}\text { Expected } \\
\text { prevalence }\end{array}$} & & \multicolumn{2}{l}{ Undiagnosed prevalence } \\
\cline { 2 - 3 } Time period & Number & $\%$ & & Number & $\%$ \\
\hline $2010 / 2011$ & 2966 & $1.6 \%$ & $3.7 \%$ & 3750 & $2.1 \%$ \\
$2009 / 2010$ & 2651 & $1.4 \%$ & & 4240 & $2.3 \%$ \\
$2008 / 2009$ & 2579 & $1.4 \%$ & & 4160 & $2.3 \%$ \\
\hline
\end{tabular}

\section{REFERENCE}

1. Bastin AJ, Starling L, Ahmed R, et al. High prevalence of undiagnosed and severe chronic obstructive pulmonary disease at first hospital admission with acute exacerbation. Chron Respir Dis 2010;7:91-7.

\section{Models of care delivery \\ P224 THE IMPACT OF IMPLEMENTING A COLLABORATIVE ANTIMICROBIAL WARD ROUND MODEL WITHIN THE RESPIRATORY DIRECTORATE OF A LARGE UNIVERSITY TEACHING HOSPITAL}

doi:10.1136/thoraxjnl-2011-201054c.224

${ }^{1} \mathrm{~S}$ E Oliver, ${ }^{1} \mathrm{E}$ Hughes, ${ }^{1} \mathrm{P}$ Lal, ${ }^{2} \mathrm{D} \mathrm{C}$ Lees, ${ }^{1} \mathrm{R}$ P Cooke, ${ }^{2} \mathrm{~B}$ Chakrabarti. ${ }^{1}$ University Hospital Aintree, Liverpool, England; ${ }^{2}$ Aintree Chest Centre, University Hospital Aintree, Liverpool, England

Introduction Clostridium difficile Infection (CDI) remains a considerable source of healthcare associated infection. A Department of Health briefing recommends all Trusts establish an antimicrobial management team (AMT) to develop an antibiotic stewardship programme aiming to reduce CDI rates through appropriate antibiotic prescribing. ${ }^{1}$ As a result, collaborative antimicrobial ward rounds were initiated in the Trust in May 2009.

Aim To study the impact of collaborative ward rounds on antibiotic prescribing within the Respiratory Directorate.

Method A weekly collaborative ward round model comprising of a Consultant microbiologist, Respiratory pharmacist and the Consultant Infection Control lead for the Respiratory Directorate was implemented across three acute respiratory wards (comprising 90 beds) in March 2011. Data were collected prospectively over a 6week period between March and May 2011 using a standardised pro-forma. Patients prescribed antibiotics were identified using the Trust's electronic prescribing system. During ward rounds, case notes and microbiology data including resistance patterns were reviewed. Treatment plans were discussed with respective clinical teams to facilitate the learning of junior medical staff. Each prescription was reviewed and recorded as appropriate if compliant with the following parameters; indication recorded, correct route, correct dose, course length documented, compliance with hospital formulary or microbiology results.

Results A total of 156 antibiotic prescriptions were reviewed during the study period; 96 (62\%) prescriptions were appropriate, 60 (38\%) required intervention. Course lengths were documented for 29 (19\%) prescriptions, $11(7 \%)$ antibiotic prescriptions were discontinued, $9(6 \%)$ antibiotic prescriptions were changed to more appropriate therapy and $6(4 \%)$ intravenous antibiotics were switched to oral therapy and $5(3 \%)$ antibiotic course lengths were extended. The defined daily doses (DDD) of antibiotics/1000 bed days over the two periods were 3544 in 2011 and 4335 in 2010 respectively (see Abstract P224 figure 1).

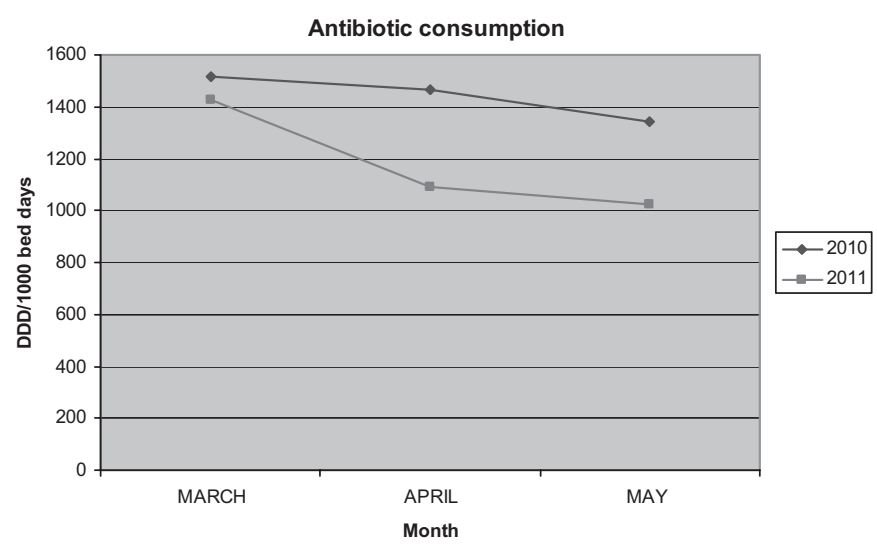

Abstract P224 Figure 1 Antibiotic consumption in DDD for the Respiratory Directorate between March and May 2011 compared with March and May 2010. 